\title{
Complete Bilateral Calcified Psoas Abscess- Rare Sequelae of Untreated Pott's Spine
}

\author{
Chaitanya Dev Pannu,' Deepika, ${ }^{2}$ Ankur Goswami,' G Vijayaraghavan' \\ 'Department of Orthopaedics, All India Institute of Medical Sciences, New Delhi, ${ }^{2}$ Department of Obs and Gynae, VMMC and \\ Safdurjung Hospitals, New Delhi, India.
}

\begin{abstract}
Although rare in the western world; psoas abscess is a frequent finding in Indian sub continent associated with Pott's spine. Untreated Pott's spine may lead to various sequelae like destruction of vertebra, kyphosis, paraplageia etc which in modern world is amenable to anti-tubercular drugs and surgical management. We report a case of untreated Pott's spine with bilateral calcified psoas abscess with kyphosis. To the best of our knowledge no such case of complete bilateral calcified psoas abscesses has been reported earlier. We want to discuss this case with relevant literature review and its influence on treatment plan.
\end{abstract}

Keywords: aminoglycoside; antistaphylococcal; psoas.

\section{INTRODUCTION}

Suppurative myositis of illiopsoas compartment results in psoas abscess which is reported to be rare. ${ }^{1}$ In developing countries where tuberculosis is prevalent secondary vertebral osteomylitis due to mycobacterium tuberculosis is a common cause of psoas abscesses. ${ }^{2}$ Psoas abscesses may develop only in $5 \%$ of the patients of vertebral tuberculosis. ${ }^{3}$ About $57 \%$ of psoas abscesses occur on the right side, $40 \%$ on the left side, and $3 \%$ bilaterally. ${ }^{4}$

We report a case of bilateral calcified psoas abscess in an untreated case of pott's spine with kyphoscoliosis. Although patchy calcifications of psoas abscess have been reported earlier ${ }^{5}$ complete calcification of the whole psoas muscle bilaterally and its implications on treatment has not been described till now.

\section{CASE REPORT}

A 25 years old female patient, with post polio residual paralysis of right hip and knee presented to our outpatient clinic with the chief complaints of deformity of back from last 10 years and back pain radiating to bilateral lower limbs from last five years. She had lowgrade fever for a period of 2-3 months at the age of 12 years but there was no history of anti-tubercular therapy. Since then she developed gradually progressive kyphotic deformity. At presentation, she was able to walk with a stooped forward posture with the help of stick on the affected side. Physical examination did not reveal any tenderness in the back (Fig.1). There was bilateral fixed flexion deformity of hip joint (right side thirty degrees and left side twenty degrees) with no tenderness in abdomen or loin and no neurological deficit in left lower limb; right side being affected by Polio with power at hip $2 / 5$ and power at knee and ankle was $3 / 5$ with intact sensations and bladder and bowel functions. Routine Blood investigations including ESR and CRP were normal. Viral marker test for HIV, HbsAg and Anti-HCV was negative and there was no history of any anti-tubercular therapy intake in the past or exposure to any close contact of active pulmonary tuberculosis. X-ray of spine showed $90^{\circ}$ kyphotic deformity in the dorsolumbar region with collapse of L1-L2 vertebra and intervening disk. Calcified bilateral paravertebral shadows were also present. Contrast enhanced MRI of whole spine did not show any tubercular activity. Bilateral psoas muscles were hypointense both in T1 and T2 weighted images. NCCT whole spine showed completely calcified psoas muscle bilaterally. Hence a probable diagnosis of healed pott's spine with bilateral calcified psoas abscess was made. We did not consider

Correspondence: Dr. Chaitanya Dev Pannu, Department of Orthopaedics, All India Institute of Medical Sciences, New Delhi, India. E-mail: cdpannu@gmail.com. 
her for correction of kyphosis due to severity of deformity associated with high risk of neurological deficit and for being associated with bilateral calcified psoas abscess. She was put on conservative management with core strengthening exercises and postural training. She was relieved of pain within six weeks.

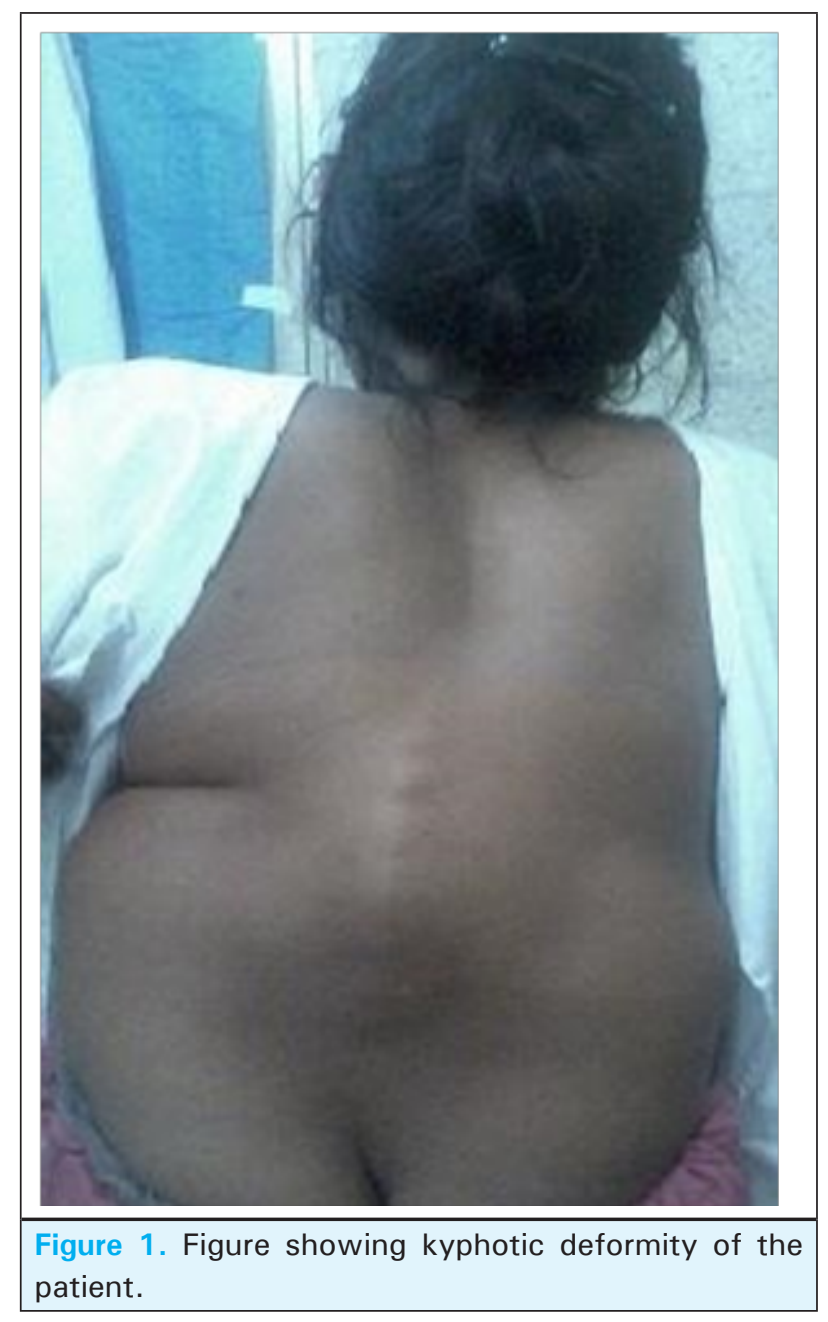

To the best of our knowledge this is the first case of bilateral fully calcified psoas abscesses reported in the English Literature. Patchy calcification is known to occur in psoas abscesses in patients with Pott's spine but it usually resolves with anti-tubercular treatment. ${ }^{5}$ Complete calcification of bilateral psoas abscesses as a sequelae of untreated Pott's spine is unknown. This changed our management and instead of doing correction of the thoracolumbar kyphosis, we had to resort to conservative management and rehabilitation.

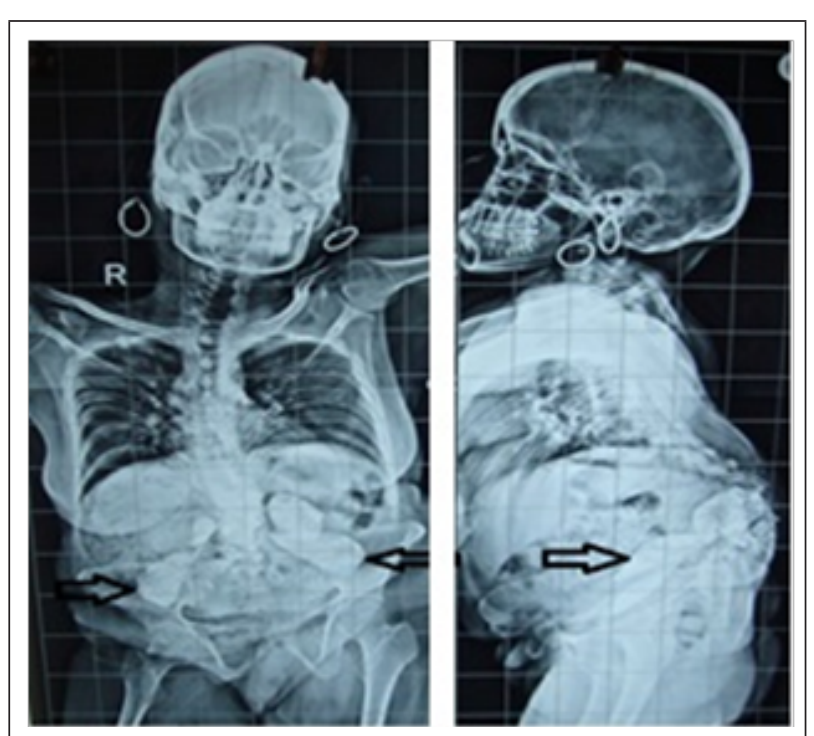

Figure 2. Calcified psoas abscess: AP and lateral views.

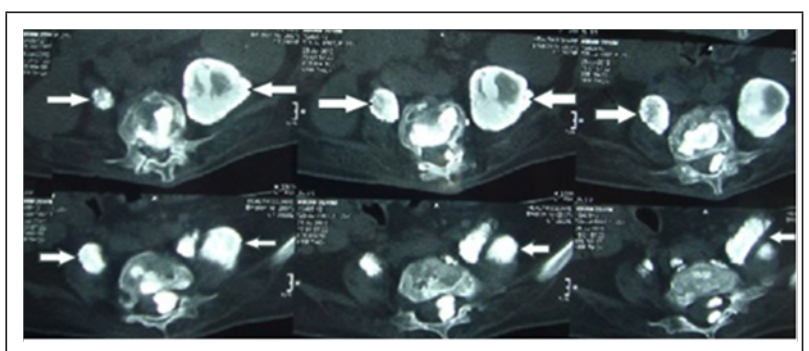

Figure 3. Bilateral calcified psoas abscess marked by white arrows on CT films.

\section{DISCUSSION}

Myenter in 1881 first described psoas abscess and referred it as psoitis. ${ }^{6}$ In western countries it is a relatively uncommon condition. Ricci et $\mathrm{al}^{7}$ reviewed 367 cases and found world-wide variations in etiology. In Asia and Africa, $99.5 \%$ of psoas abscesses are primary, whereas $17.7 \%$ and $61 \%$ are primary in Europe and North America, respectively. ${ }^{7}$ It is found more commonly in young patients as compared with the elderly. ${ }^{8}$ Males are more commonly affected than females. ${ }^{9}$

When the source of infection is unknown it is said to be primary and presumed to arise by hematogenous spread, or secondary to an adjacent retroperitoneal or intraabdominal infection. ${ }^{1}$ Risk factors of the primary psoas abscess are history of diabetes, injection drug use, alcoholism, AIDS, renal failure, hematologic malignancy, immunosuppression or malnutrition. Most common causative organism is Staphylococcus aureus $(\geq 88 \%)$, followed by Escherichia coli and Streptococcus. ${ }^{7}$ E. Coli and bacteroides and other constituents of the mixed enteric flora are the causative agents of the secondary psoas abscess. ${ }^{7}$ In 
developing countries psoas abscess is most commonly caused secondarily to mycobacterium tuberculosis infection of the spine. ${ }^{7}$ Secondary psoas abscess can occur in conditions like Crohn's disease, diverticulitis, appendicitis, colorectal cancer, urinary tract infection, vertebral osteomyelitis, mycotic abdominal aortic aneurysm, endocarditis, and history of instrumentation in or around the spine. ${ }^{1}$ Clinically symptoms are usually non-specific. History of fever, chills, loss of apettite, night sweats, malaise, discomfort and weight loss may be present. Usually back pain is most common reported symptom. Patient may complaint of pain in abdomen, groin, hip, or knee. On examination patient may have fever, tachycardia, and generalized wasted appearance. Tenderness in acute cases and a mass in chronic cases can be present in the lower abdomen, groin, back or thigh. Movements of the ipsilateral hip may be painful in acute stages due to psoas spasm. There may be limp on the affected side or lateral curvature (scoliosis) of the spine and eventually flexion deformity of the hip develops. Diagnosis is often delayed because of the non specific complaints in acute stages which resembles most commonly with vertebral osteomyelitis, lumbar strain, and abdominal or urologic disorders. A thorough medical history and meticulous physical examination is the cornerstone for the diagnosis of psoas abscess presenting with usual clinical finding.

Laboratory tests helpful for diagnosis are complete blood count ( $C B C$ ) with differential count, erythrocyte sedimentation rate (ESR), C-Reactive protein (CRP) and blood and urine cultures. In X-rays plain abdominal x-rays are usually sufficient but $x$-rays with a barium enema or taken after injection of a radiopaque dye (intravenous pyelogram), and an upper gastrointestinal series may be useful in diagnosis. The most significant feature in plain radiograph of abdomen is loss of definition of the psoas muscle. Sometimes abnormal calcification and gas in the soft tissues may also be visible. USG and CT can be used for both diagnosis and therapeutic drainages.

\section{Treatment}

The first step of management is to ascertain the nature (primary / secondary). As most common causative agent of the primary psoas abscesses is staphylococcus aureus, broad spectrum antistaphylococcal antibiotics should be started before the culture results and in secondary iliopsoas abscess antibiotics like clindamycin, antistaphylococcal penicillin, and an aminoglycoside are started. In Indian subcontinent as the incidence of psoas abscesses secondary to tubercular vertebral osteomyelitis is very high; after complete hemogram, ESR, CRP, plain radiographs and confirming the features of tubercular osteomyelitis on MRI scans, antitubercular drugs are started. Indications for surgical intervention are present only in selected patients when disease progresses even on medical treatment or spinal cord compression causing neurological deficit in Pott's spine. Aspiration of abscesses is performed to confirm the diagnosis and to know the antibiotic sensitivity of the organism in unresponsive cases. We do not aspirate psoas abscess routinely. We consider that anti-tuberculous treatment would be able to cause subsidence of abscesses without the need for surgical drainage.

Abscess can be drained through CT or ultrasound guided percutaneous drainage or open surgical drainage. Single time ultrasound guided percutaneous drainage is our method of choice. Mueller et al reported first application of percutaneous drainage in iliopsoas abscesses in 1984. Surgical drainage may be ideal for patients with underlying Crohn's disease or other gastrointestinal diseases. In these patients a single sitting surgical intervention involving resection anastomosis of bowel and drainage of psoas abscess is desirable. ${ }^{11}$ Deep and multiloculated abscesses are not amenable to percutaneous drainage. Parameters most often used to monitor the recovery are the total leukocyte count, serial ESR and CRP and serial abdominal USG and MRI scans to look for volume and distribution of the abscesses ${ }^{11}$.

\section{Prognosis}

Primary psoas abscess has a better prognosis, the mortality rate being only $2.4 \%$. Secondary psoas abscess has a mortality rate of $18.9 \% .^{10}$ The major cause of death is delayed or inadequate therapy.

\section{ACKNOWLEDGEMENTS}

The author would like to acknowledge the patient who willingly participated.

\section{REFERENCES}

1. Taiwo B, et al. Psoas abscess: a primer for the internist. South. Med. J. 2001;94:2-5.

2. Mückley T, Schütz T, Kirschner M, Potulski M, Hofmann G, Bühren V, et al. Psoas abscess: the spine as a primary source of infection. Spine. 2003;28:E106-13.

3. Huang JJ, Ruaan MK, Lan RR, Wang MC. Acute pyogenic iliopsoas abscess in Taiwan: clinical features, diagnosis, treatments and outcome. J. Infect. 2000;40:248-55.

4. Thongngarm T, McMurray RW. Primary psoa abscess. Ann. Rheum. Dis. 2001;60:173-4. 
Pannu et al. Complete Bilateral Calcified Psoas Abscess- Rare Sequelae of Untreated Pott's Spine

5. Gazzaz M, Elabbadi N, Alifdal M, Jidal M, Bellakhdar F. Calcified tuberculous abscess of the psoas muscle. Apropos of a case Acta Neurol Belg. 1995 Dec;95(4):247-8.

6. Mynter H. Acute psoitis. Buffalo Med Surg J. 1881;21:202-10.

7. Ricci MA, Rose FB, Meyer KK. Pyogenic psoas abscess: worldwide variations in aetiology. World J Surg. 1986; 10: 834-43.

8. Gruenwald I, Abrahamson J, Cohen O. Psoas abscess: case report and review of the literature.J Urol. 1992;147:1624-6.
9. Paley M, Sidhu PS, Evans RA, Karani JB. Retroperitoneal collections-aetiology and radiological implications.Clin Radiol. 1997;52:290-4.

10. Qureshi NH, O'Brien DP, Allcutt DA. Psoas abscess secondary to discitis: a case report of conservative management. J Spinal Disord. 2000;13:73-6.

11. Procaccino JA, Lavery IC, Fazio VW, Oakley JR. Psoas abscess: difficulties encountered. Dis Colon Rectum. 1992: 35:513-514. 